\title{
Different Types of Statins and All-Cause Mortality during Anticoagulation for Venous Thromboembolism: Validation Study from RIETE Registry
}

\author{
Carmine Siniscalchi ${ }^{10}$ José M. Suriñach ${ }^{2}$ Adriana Visonà ${ }^{3}$ José L. Fernández-Reyes ${ }^{4}$ \\ Covadonga Gómez-Cuervo $^{5}{ }^{\circledR}$ Peter Verhamme $^{6} \quad$ Pablo J. Marchena $^{7}$ Dominique Farge-Bancel ${ }^{8}$ \\ Jorge Moisés $^{90}$ Manuel Monreal ${ }^{10}$ and the RIETE Investigators*
}

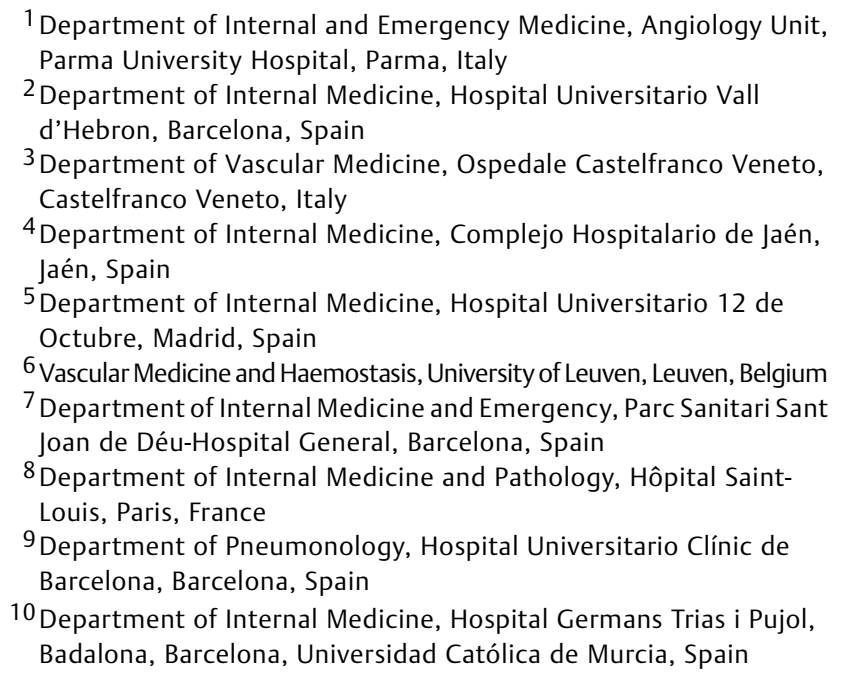

Address for correspondence Carmine Siniscalchi, MD, PhD, Department of Internal and Emergency Medicine, Angiology Unit, Parma University Hospital, Parma 43125, Italy (e-mail: csiniscalchi84@gmail.com).

TH Open 2020;4:e236-e244.

\footnotetext{
* A complete list of RIETE investigators is given in the -Supplementary Material.
}

received

March 23, 2020

accepted after revision

August 11, 2020
Introduction We previously reported that during the course of anticoagulation for venous thromboembolism (VTE) patients using statins were at a lower risk to die than nonusers.

Methods We used the Registro Informatizado Enfermedad TromboEmbólica (RIETE) registry to validate our previous findings in a subsequent cohort of patients and to compare the risk of death according to the use of different types of statins.

Results From January 2018 to December 2019, 19,557 patients with VTE were recruited in RIETE. Of them, 4,065 (21\%) were using statins (simvastatin, 1,406; atorvastatin, 1,328; rosuvastatin, 246; and others, 1 ,085). During anticoagulation (192 vs. 182 days, for statin and no statin users respectively), 500 patients developed a VTE recurrence, 519 suffered major bleeding, and 1,632 died (fatal pulmonary embolism [PE], 88 and fatal bleeding, 78). On multivariable analysis, statin users were at a lower risk to die (hazard ratio $[\mathrm{HR}]=0.68 ; 95 \%$ confidence interval $[\mathrm{Cl}]: 0.59-0.79$ ) than 
nonusers. When separately analyzing the drugs, on multivariable analysis, patients using simvastatin ( $\mathrm{HR}=0.64 ; 95 \% \mathrm{Cl}: 0.52-0.80)$, atorvastatin ( $\mathrm{HR} 0.72 ; 95 \% \mathrm{Cl}$ : $0.58-0.89$ ), or other statins ( $\mathrm{HR}=0.67 ; 95 \% \mathrm{Cl}: 0.52-0.87$ ) were at a lower risk to die than nonusers. For those using rosuvastatin, difference was not statistically significant ( $\mathrm{HR}=0.77 ; 95 \% \mathrm{Cl}: 0.50-1.19)$, maybe due to the sample size.

Conclusion Our data validate previous findings and confirm that VTE patients using statins at baseline are at a lower risk to die than nonusers. No statistically differences were found according to type of statins.

\section{Introduction}

The impact of statins on survival in patients with coronary, cerebrovascular, or peripheral artery disease has been extensively studied, ${ }^{1-6}$ but only few studies have evaluated their influence on mortality in patients receiving anticoagulation for venous thromboembolism (VTE). ${ }^{7-9}$ Several prior studies suggest a lower rate of VTE recurrences in statin users during anticoagulation for VTE. The JUPITER study reported a $43 \%$ reduction with the use of rosuvastatin ${ }^{7}$ but did not provide data on mortality. ${ }^{7}$ A recent meta-analysis and metaregression of randomized controlled trials ${ }^{8,9}$ demonstrated that statin therapy affects coagulation factors and thrombin generation, but its relationship whit the risk to die during anticoagulation in statin users has not been consistently studied. One study found that patients with acute pulmonary embolism (PE) who were using statins at baseline had half the risk to die than nonusers, ${ }^{10}$ and a case-control study also found that statin users presenting with VTE were at a lower risk for death than nonusers. ${ }^{11}$ However, both studies were based on population registries of records linked with hospital discharge records where the diagnosis of VTE is based on diagnostic codes, and mortality was a secondary outcome. In a recent Registro Informatizado Enfermedad TromboEmbólica (RIETE) study that included 32,062 patients with a first episode of VTE, we observed that those using statins ( $22 \%$ of the whole cohort) demonstrated a $38 \%$ lower mortality compared with nonusers. ${ }^{12}$

The RIETE registry is an ongoing, multicenter, observational registry of consecutive patients with objectively confirmed acute VTE (ClinicalTrials.gov identifier: NCT02832245). Data from this registry have been used to evaluate outcomes after acute VTE such as the frequency of recurrent VTE, major bleeding or mortality, and risk factors for these outcomes. ${ }^{13-18}$ The rationale and methodology of RIETE have been published elsewhere. ${ }^{19}$ In the current analysis, we aimed to validate our prior observations in a subsequent cohort of patients with VTE and to compare the mortality risk according to the type of statins.

\section{Methods}

\section{Inclusion Criteria}

Consecutive patients with acute, symptomatic deep vein thrombosis (DVT) or PE confirmed by objective tests (compression ultrasonography or contrast venography for DVT; helical computed tomography [CT] scan of the chest, ventila- tion-perfusion lung scintigraphy, or angiography for PE) were enrolled in RIETE. Patients were excluded if they were currently participating in a blinded/double-blind therapeutic clinical trial. All patients (or their relatives) provided writtenor oral-informed consent for participation in the registry, in accordance with local ethics committee requirements.

\section{Study Design}

For this study, only patients with available information on the use of statins at baseline and who were not previously included in the prior study were considered. Thus, we included all patients with acute symptomatic VTE recruited from February 2018 to December 2019. The major study outcome was all-cause mortality. Secondary outcomes were fatal PE and fatal bleeding. Bleeding events were classified as major if they were overt and required a transfusion of 2 units of blood or more, or were retroperitoneal, spinal, or intracranial. Fatal PE, in the absence of autopsy, was defined as any death appearing $<10$ days after PE diagnosis, in the absence of any alternative cause of death. Fatal bleeding was defined as any death occurring $<10$ days after a major bleeding episode, in the absence of any alternative cause of death.

\section{Study Variables}

The following parameters are recorded in RIETE: patient's baseline characteristics; clinical status including any coexisting or underlying conditions such as chronic heart or lung disease, recent major bleeding, anemia, or renal insufficiency; risk factors for VTE; the treatment received upon VTE diagnosis; concomitant drugs; and the outcomes during the course of therapy. Immobilized patients were defined as nonsurgical patients who had been immobilized (i.e., total bed rest with or without bathroom privileges) for $\geq 4$ days in the 2-month period prior to VTE diagnosis. Surgical patients were defined as those who had undergone an operation in the 2 months prior to VTE. Active cancer was defined as newly diagnosed cancer ( $<3$ months before) or when receiving antineoplastic treatment of any type (i.e., surgery, chemotherapy, radiotherapy, hormonal, support therapy, or combined therapies). Recent bleeding was defined as major bleeding $<30$ days prior to VTE. Anemia was defined as hemoglobin levels $<13 \mathrm{~g} / \mathrm{dL}$ for men and $<12 \mathrm{~g} / \mathrm{dL}$ for women.

\section{Treatment and Follow-up}

Patients were managed according to the clinical practice of each participating hospital (i.e., there was no standardization 
of treatment). The type, dose, and duration of anticoagulant therapy were recorded. After VTE diagnosis, all patients were followed-up in the outpatient clinic for at least 3 months, and thereafter as long as possible. During each visit, any signs or symptoms suggesting VTE recurrences or bleeding complications were noted. Each episode of clinically suspected recurrent VTE was investigated by repeat compression ultrasonography, lung scanning, helical CT scan, or pulmonary angiography as appropriate. The RIETE investigators assessed mortality using medical record review, and proxy interviews when necessary. Most outcomes were classified as reported by the clinical centers. However, if staff at the coordinating center were uncertain how to classify a reported outcome, the event was reviewed by a central adjudicating committee $(<10 \%$ of events).

\section{Statistical Analysis}

Categorical variables were compared using the Chi-square test (two-sided) and Fisher's exact test (two-sided). Continuous variables were compared using Student's $t$-test. Incidence rates were calculated as events per 100 patient-years of follow-up and compared between patients receiving and those not receiving statins at baseline, using the hazard ratios (HRs) and corresponding 95\% confidence intervals (CIs). We compared the cumulative mortality rates between statin users and nonusers, and also according to the use of different types of statins with time-to-event methods. Mortality was assessed with Cox's proportional hazard model. Time zero was the date of VTE and censoring occurred at the time of last follow-up. Covariates included in the adjusted model were those for which a statistically significant difference (a threshold $p$-value of 0.1 was set to assess significance of differences) was found, and a backward selection was used for the covariate selection in the multivariable model. Statistical analyses were conducted with SPSS for Windows Release (version 20, SPSS Inc. Chicago, Illinois, United States).

\section{Results}

From January 2018 to December 2019, 19,557 patients with VTE were recruited in RIETE (-Fig. 1). Of them, 4,065 (21\%) were using statins at baseline (simvastatin, 1,406 ; atorvastatin, 1,328; rosuvastatin, 246; and other statins, 1,085). Compared with nonusers, statin users were 9 years older and were more likely to have comorbidities such as chronic heart failure, lung disease, hypertension, diabetes, prior myocardial infarction, prior ischemic stroke, peripheral artery disease, anemia, or renal insufficiency (-Table 1). Statin users were also more likely to be using antiplatelet agents or corticosteroids at baseline and had lower levels of total- and low-density lipoprotein (LDL)-cholesterol at baseline. Similar rates of active cancer were found in both subgroups.

Most patients in both subgroups were initially treated with low molecular weight heparin (LMWH), at similar daily doses (-Table 2). Among the remaining patients, statin users were less likely to receive direct oral anticoagulants (DOACs) than nonusers. For long-term therapy, statin users were more likely to receive vitamin-K antagonists and less likely

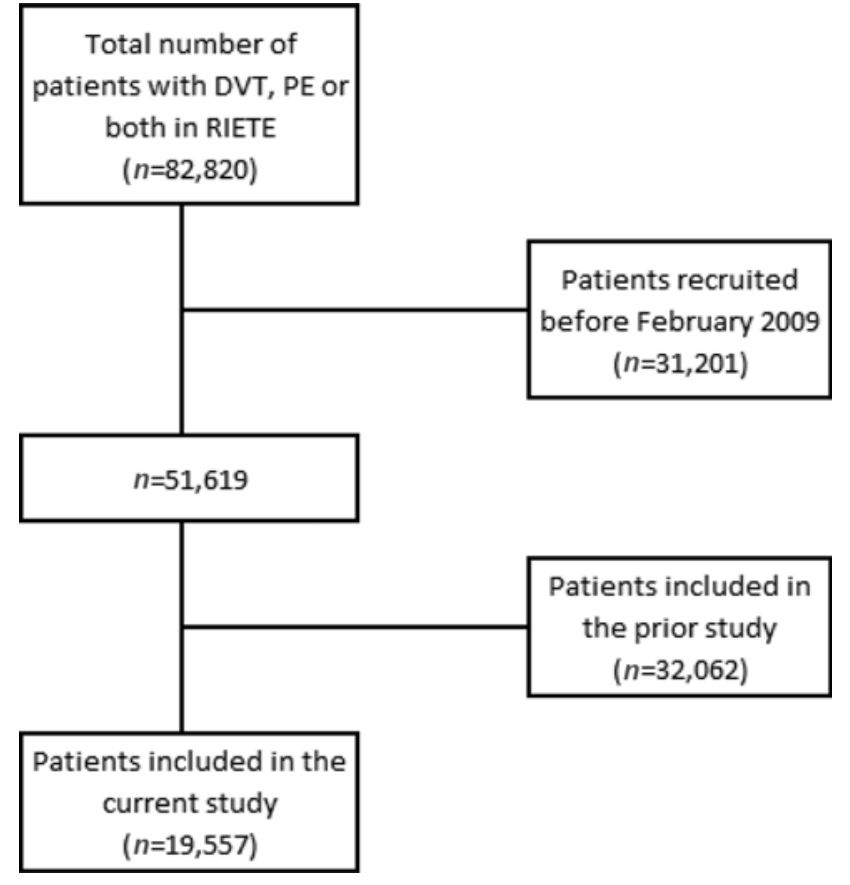

Fig. 1 Patients with VTE who were recruited in Registro Informatizado Enfermedad TromboEmbólica (RIETE). DVT, deep vein thrombosis; PE, pulmonāry embōlism.

to receive DOACs. Among all, 63 patients did not receive anticoagulant therapy, and were excluded from the analysis. Median duration of anticoagulant therapy was slightly longer in statin users: 192 (interquartile range [IQR]: 104-376 days) versus 182 (IQR: 102-345) days, respectively.

During the course of anticoagulant therapy, 500 patients developed VTE recurrences, 519 had major bleeding and 1,632 died (fatal PE, 88 and fatal bleeding 78). In nonadjusted analyses, statin users had a significantly lower mortality rate ( $\mathrm{HR}=0.80 ; 95 \% \mathrm{CI}: 0.71-0.91)$, a nonsignificantly lower rate of VTE recurrences $(\mathrm{HR}=0.84 ; 95 \% \mathrm{CI}$ : 0.67-1.04), and a higher rate of major bleeding $(\mathrm{HR}=1.27 ; 95 \% \mathrm{CI}: 1.04-1.54)$ than nonusers (-Table 3). Statin users had a nonsignificant lower rates of fatal $\mathrm{PE}(\mathrm{HR}=0.67 ; 95 \% \mathrm{CI}: 0.37-1.17)$ and a similar rate of fatal bleeding $(\mathrm{HR}=1.07 ; 95 \% \mathrm{CI}$ : 0.62-1.79; - Table 4$)$.

Compared with nonstatin users, patients using simvastatin $(\mathrm{HR}=0.72 ; 95 \% \mathrm{Cl}: 0.58-0.88)$ or other statins $(\mathrm{HR}=0.70$; 95\% CI: $0.55-0.89$ ) had a lower mortality rate, but there were no significant differences in those using atorvastatin $(\mathrm{HR}=1.00 ; 95 \% \mathrm{CI}: 0.83-1.21)$ or rosuvastatin $(\mathrm{HR}=1.10$; 95\% CI: 0.73-1.66; - Table 5).

On multivariable analysis (after adjusting for patients' age, body weight, chronic lung or heart disease, diabetes, prior artery disease, recent major bleeding, risk factors for VTE, initial VTE presentation, anemia, platelet count, renal function, and concomitant therapy with antiplatelet agents or corticosteroids), statin users had a significantly lower risk to die ( $\mathrm{HR}=0.68 ; 95 \% \mathrm{CI}$ : 0.59-0.79) compared with nonusers. Concerning the different statins, patients using simvastatin $(\mathrm{HR}=0.64 ; 95 \% \mathrm{Cl}: 0.52-0.80)$, atorvastatin $(\mathrm{HR}=0.72 ; 95 \%$ CI: $0.58-0.89)$, or other statins ( $\mathrm{HR}=0.67 ; 95 \% \mathrm{CI}: 0.52-0.87$ ) were at a lower risk to die than nonusers. Patients using 
All-Cause Mortality during Anticoagulation for VTE Siniscalchi et al. e239

Table 1 Clinical characteristics in 19,557 patients with VTE, according to use of statins at baseline

\begin{tabular}{|c|c|c|c|}
\hline & Statins users & Non-users & Odds ratio $(95 \% \mathrm{Cl})$ \\
\hline Patients $(n)$ & 4,065 & 15,492 & \\
\hline \multicolumn{4}{|l|}{ Clinical characteristics } \\
\hline $\begin{array}{l}\text { Age }(y) \\
\text { Mean } \pm \text { SD }\end{array}$ & $73 \pm 11$ & $64 \pm 18^{c}$ & $<0.0001$ \\
\hline Male & $2,013(50)$ & $7,800(50)$ & $0.97(0.90-1.04)$ \\
\hline $\begin{array}{l}\text { Body weight }(\mathrm{kg}) \\
\text { Mean } \pm \mathrm{SD}\end{array}$ & $78 \pm 15$ & $77 \pm 17^{c}$ & $<0.0001$ \\
\hline \multicolumn{4}{|l|}{ Comorbidities } \\
\hline Chronic lung disease & $605(15)$ & $1,626(10)^{c}$ & $1.49(1.35-1.65)$ \\
\hline Chronic heart failure & $441(11)$ & $818(5.3)^{c}$ & $2.18(1.93-2.46)$ \\
\hline Recent major bleeding & $91(2.2)$ & $331(2.1)$ & $1.05(0.83-1.33)$ \\
\hline Hypertension & 2,995 (74) & $5,669(37)^{c}$ & $4.85(4.49-5.24)$ \\
\hline Diabetes & $1,151(28)$ & $1,620(10)^{c}$ & $3.38(3.11-3.68)$ \\
\hline Current smoking & $387(9.5)$ & $2,053(13)^{c}$ & $0.69(0.61-0.77)$ \\
\hline Prior myocardial infarction & $700(17)$ & $479(3.6)^{c}$ & $5.60(4.96-6.33)$ \\
\hline Prior ischemic stroke & $480(12)$ & $578(4.4)^{c}$ & $2.96(2.61-3.36)$ \\
\hline Peripheral artery disease & $298(7.5)$ & $357(2.7)^{c}$ & $2.87(2.45-3.37)$ \\
\hline \multicolumn{4}{|l|}{ Concomitant therapies } \\
\hline Antiplatelets & $1,463(36)$ & $1,747(11)^{c}$ & $4.42(4.08-4.80)$ \\
\hline Corticosteroids & $396(9.7)$ & $1,241(8.0)^{c}$ & $1.24(1.10-1.40)$ \\
\hline \multicolumn{4}{|l|}{ Risk factors for VTE } \\
\hline Immobilization $\geq 4$ days & 777 (19) & $2,800(18)$ & $1.07(0.98-1.17)$ \\
\hline Active cancer & $607(15)$ & $2,536(16)^{a}$ & $0.90(0.81-0.99)$ \\
\hline Surgery & $379(9.3)$ & $1,475(9.5)$ & $0.98(0.87-1.10)$ \\
\hline Estrogen use & $82(2.0)$ & $863(5.6)^{c}$ & $0.35(0.28-0.44)$ \\
\hline Pregnancy or postpartum & $4(0.10)$ & $176(1.1)^{c}$ & $0.09(0.03-0.23)$ \\
\hline None of the above & $2,461(61)$ & $8,663(56)^{c}$ & $1.21(1.13-1.30)$ \\
\hline \multicolumn{4}{|l|}{ Initial VTE presentation } \\
\hline Pulmonary embolism & $2,403(59)$ & $8,511(55 \%)^{c}$ & $1.19(1.11-1.27)$ \\
\hline \multicolumn{4}{|l|}{ In patients with $\mathrm{PE}$} \\
\hline SBP levels $<100 \mathrm{~mm} \mathrm{Hg}$ & $158(6.6)$ & $612(7.2)$ & $0.91(0.76-1.09)$ \\
\hline Heart rate $>110$ bpm & $330(14)$ & $1,253(15)$ & $0.90(0.79-1.03)$ \\
\hline Sat $\mathrm{O}_{2}$ levels $<90 \%$ & $365(30)$ & $1,052(27)^{a}$ & $1.16(1.00-1.33)$ \\
\hline \multicolumn{4}{|l|}{ Blood tests } \\
\hline Anemia & $1,344(33)$ & $4,848(31 \%)^{a}$ & $1.08(1.01-1.17)$ \\
\hline Platelet count $<100,000 / \mu \mathrm{L}$ & $105(2.6)$ & $370(2.4)$ & $1.08(0.87-1.35)$ \\
\hline $\mathrm{CrCl}$ levels $<50 \mathrm{~mL} / \mathrm{min}$ & $1,075(26)$ & $2,771(18)^{c}$ & $1.65(1.52-1.79)$ \\
\hline $\begin{array}{l}\text { Cholesterol levels }(\mathrm{mg} / \mathrm{dL}) \\
\text { Mean } \pm \text { SD }\end{array}$ & $170 \pm 43$ & $182 \pm 67^{c}$ & 0.000 \\
\hline $\begin{array}{l}\text { LDL-cholesterol levels (mg/dL) } \\
\text { Mean } \pm \text { SD }\end{array}$ & $101 \pm 85$ & $120 \pm 186^{c}$ & 0.001 \\
\hline
\end{tabular}

Abbreviations: $\mathrm{Cl}$, confidence intervals; $\mathrm{CrCl}$, creatinine clearance; LDL, low-density lipoprotein; PE, pulmonary embolism; SD, standard deviation; VTE, venous thromboembolism.

Notes: Age is presented as mean ( \pm standard deviation). Other numbers presented as $n(\%)$.

Differences between statin users and nonusers: ${ }^{\mathrm{a}} p<0.05$; ${ }^{\mathrm{b}} p<0.01 ;{ }^{\mathrm{c}} p<0.001$. 
e240 All-Cause Mortality during Anticoagulation for VTE Siniscalchi et al.

Table 2 Treatment strategies

\begin{tabular}{|c|c|c|c|}
\hline & Statins users & Non users & OR $(95 \% \mathrm{Cl})$ \\
\hline Patients $(n)$ & 4,065 & 15,492 & \\
\hline \multicolumn{4}{|l|}{ Initial therapy } \\
\hline LMWH & $3,522(87)$ & $12,865(83)^{c}$ & $1.32(1.20-1.46)$ \\
\hline Mean LMWH doses (IU/kg/day) & $168 \pm 45$ & $169 \pm 44$ & 0.085 \\
\hline Unfractionated heparin & $167(4.1)$ & $759(4.9)^{\mathrm{a}}$ & $0.83(0.70-0.99)$ \\
\hline Fondaparinux & $77(1.9)$ & $443(2.9)^{c}$ & $0.66(0.51-0.84)$ \\
\hline Direct oral anticoagulants & $171(4.2)$ & $957(6.2)^{c}$ & $0.67(0.56-0.79)$ \\
\hline Thrombolytics & $57(1.4)$ & $210(1.4)$ & $1.03(0.77-1.39)$ \\
\hline No anticoagulant therapy & $17(0.42)$ & $46(0.30)$ & $1.41(0.81-2.46)$ \\
\hline Vena cava filter & $120(3.0)$ & $424(2.7)$ & $1.08(0.88-1.33)$ \\
\hline \multicolumn{4}{|l|}{ Long-term therapy } \\
\hline Vitamin-K antagonists & $2,112(52)$ & $7,325(47)^{c}$ & $1.21(1.13-1.29)$ \\
\hline LMWH & $1,167(29)$ & $4,553(29)$ & $0.97(0.90-1.04)$ \\
\hline Mean LMWH doses (IU/kg/day) & $149 \pm 44$ & $152 \pm 45^{\mathrm{a}}$ & 0.030 \\
\hline Fondaparinux & $19(0.47)$ & $129(0.83)^{\mathrm{a}}$ & $0.56(0.35-0.91)$ \\
\hline Direct oral anticoagulants & $664(16)$ & $2,998(19)^{c}$ & $0.81(0.74-0.89)$ \\
\hline \multicolumn{4}{|l|}{ Duration of anticoagulant therapy } \\
\hline Mean days $( \pm S D)$ & $313 \pm 352$ & $292 \pm 364^{b}$ & 0.001 \\
\hline Median days (IQR) & $192(104-376)$ & $182(102-345)$ & 0.000 \\
\hline Over 6 months & $2,193(54)$ & $7,848(51)^{c}$ & $1.14(1.06-1.22)$ \\
\hline Over 12 months & $1,087(27)$ & $3,515(23)^{c}$ & $1.24(1.15-1.35)$ \\
\hline
\end{tabular}

Abbreviations: $\mathrm{Cl}$, confidence intervals; IQR, interquartile range; IU, international units; LMWH, low molecular weight heparin; OR, odds ratio; SD, standard deviation.

Notes: Values are presented as mean ( \pm standard deviation), median (IQR), and $n(\%)$.

Differences between statin users and nonusers: ${ }^{\mathrm{a}} p<0.05$; ${ }^{\mathrm{b}} p<0.01 ;{ }^{\mathrm{c}} p<0.001$.

rosuvastatin had a nonsignificantly lower risk ( $\mathrm{HR}=0.77 ; 95 \%$ CI: 0.50-1.19), as shown in - Table 5 and - Fig. 2.

\section{Discussion}

Our data confirm that VTE patients using statins at baseline are at a lower risk to die than nonusers, as previously reported. One out of four patients (26\%) in our cohort were using statins at baseline, and during anticoagulation, they had a $20 \%$ lower rate of mortality rate despite being older and more likely to have comorbidities. Multivariable adjusted analyses confirmed that this decrease was independent from several potential confounders, and didn't show differences between types of statins.

Statins are widely used in patients with cardiovascular risk factors ${ }^{20}$ and their influence on survival in these patients has been largely reported. ${ }^{1-5}$ Few studies investigated their influence on mortality in patients receiving anticoagulation for VTE. In our previous study, we found that statin users $(n=7,085)$ had a $38 \%$ reduction in all-cause mortality compared with nonusers (adjusted $\mathrm{HR}=0.62 ; 95 \% \mathrm{CI}$ : $0.48-0.79) .{ }^{12}$ The current study validates and confirms these results with a similar decrease (adjusted $\mathrm{HR}=0.68 ; 95 \% \mathrm{CI}$ : 0.59-0.79). Our findings are based on a cohort of patients with symptomatic, objectively proven diagnosis of VTE, not on an administrative database where the diagnosis of VTE was based on less specific diagnostic codes and hospital discharge records as in some other studies on this topic. ${ }^{10,11}$

Interestingly however, we failed to find differences between types of statins. In particular, we failed to find differences for those statins that lower LDL-C or total cholesterol levels more potently. Although we cannot provide a biologically plausible explanation, these findings might be due to two reasons. First, the observed lower mortality rate is not due to the effect of statins on LDL-C or total cholesterol levels, but rather to other "pleiotropic" mechanisms, such as platelet inhibition, reduction of inflammation, reduction of C-reactive protein, increased production of nitric oxide, or downregulation of the coagulation cascade. ${ }^{6,21}$ This is supported by meta-analysis and metaregression of randomized controlled trials ${ }^{8}$ and randomized clinical trial (START) ${ }^{9}$ and several clinical studies demonstrating that statin therapy either with rosuvastatin, ${ }^{21}$ simvastatin, $^{22}$ atorvastatin, ${ }^{23,24}$ or cerivastatin ${ }^{25}$ affects coagulation factors and thrombin generation. In our study, no differences in levels of total or LDL cholesterol at baseline were observed and this can be in line with this hypothesis. Second, also in patients with cardiovascular risk factors, statin use may decrease mortality not only through their lipid-lowering effect 
Table 3 Clinical outcomes during the course of anticoagulant therapy

\begin{tabular}{|c|c|c|c|c|c|}
\hline & \multicolumn{2}{|c|}{ Statins users } & \multicolumn{2}{|c|}{ Nonusers } & \multirow{2}{*}{$\begin{array}{l}\text { Hazard ratio } \\
(95 \% \mathrm{Cl})\end{array}$} \\
\hline & $n$ & $\begin{array}{l}\text { Events per } \\
100 \text { patient-years }\end{array}$ & $n$ & $\begin{array}{l}\text { Events per } \\
100 \text { patient-years }\end{array}$ & \\
\hline Patients $(n)$ & \multicolumn{2}{|c|}{4,065} & \multicolumn{2}{|c|}{15,492} & \\
\hline Recurrent PE & 46 & $1.34(0.99-1.77)$ & 185 & $1.51(1.30-1.74)$ & $0.89(0.64-1.22)$ \\
\hline Recurrent DVT & 53 & $1.55(1.18-2.02)$ & 238 & $1.96(1.72-2.22)$ & $0.79(0.59-1.06)$ \\
\hline Recurrent VTE & 95 & $2.81(2.29-3.42)$ & 405 & $3.36(3.05-3.70)$ & $0.84(0.67-1.04)$ \\
\hline Major bleeding & 136 & $3.97(3.34-4.68)$ & 383 & $3.13(2.83-3.46)^{a}$ & $1.27(1.04-1.54)$ \\
\hline \multicolumn{6}{|l|}{ Site of major bleeding } \\
\hline Gastrointestinal & 47 & $1.36(1.01-1.79)$ & 139 & $1.13(0.95-1.33)$ & $1.20(0.86-1.67)$ \\
\hline Hematoma & 24 & $0.69(0.45-1.01)$ & 86 & $0.70(0.56-0.86)$ & $0.99(0.62-1.55)$ \\
\hline Intracranial & 29 & $0.84(0.57-1.19)$ & 68 & $0.55(0.43-0.69)$ & $1.52(0.97-2.33)$ \\
\hline Myocardial infarction & 8 & $0.23(0.11-0.44)$ & 19 & $0.15(0.10-0.24)$ & $1.50(0.62-3.37)$ \\
\hline Ischemic stroke & 22 & $0.64(0.41-0.95)$ & 55 & $0.45(0.34-0.58)$ & $1.43(0.86-2.32)$ \\
\hline Death & 300 & $8.64(7.70-9.66)$ & 1,332 & $10.8(10.2-11.4)^{c}$ & $0.80(0.71-0.91)$ \\
\hline \multicolumn{6}{|l|}{ Causes of death } \\
\hline Pulmonary embolism & 14 & $0.40(0.23-0.66)$ & 74 & $0.60(0.47-0.75)$ & $0.67(0.37-1.17)$ \\
\hline Bleeding & 18 & $0.52(0.32-0.80)$ & 60 & $0.48(0.37-0.62)$ & $1.07(0.62-1.79)$ \\
\hline Respiratory failure & 16 & $0.46(0.27-0.73)$ & 96 & $0.78(0.63-0.94)^{a}$ & $0.59(0.34-0.99)$ \\
\hline Sudden, unexpected & 7 & $0.20(0.09-0.40)$ & 21 & $0.17(0.11-0.25)$ & $1.19(0.47-2.72)$ \\
\hline Disseminated malignancy & 125 & $3.60(3.01-4.27)$ & 548 & $4.43(4.07-4.81)^{a}$ & $0.81(0.67-0.98)$ \\
\hline Infection & 22 & $0.63(0.41-0.94)$ & 98 & $0.79(0.65-0.96)$ & $0.80(0.49-1.25)$ \\
\hline Multiorganic failure & 17 & $0.49(0.29-0.77)$ & 85 & $0.69(0.55-0.85)$ & $0.71(0.41-1.18)$ \\
\hline Heart failure & 15 & $0.43(0.25-0.70)$ & 51 & $0.41(0.31-0.54)$ & $1.05(0.57-1.83)$ \\
\hline Bronchoaspiration & 6 & $0.17(0.07-0.36)$ & 38 & $0.31(0.22-0.42)$ & $0.56(0.22-1.27)$ \\
\hline Ischemic stroke & 4 & $0.12(0.04-0.28)$ & 8 & $0.06(0.03-0.12)$ & $1.78(0.47-5.89)$ \\
\hline Myocardial infarction & 2 & $0.06(0.01-0.19)$ & 7 & $0.06(0.02-0.11)$ & $1.02(0.14-4.57)$ \\
\hline
\end{tabular}

Abbreviations: $\mathrm{Cl}$, confidence intervals; DVT, deep vein thrombosis; PE, pulmonary embolism.

Note: Differences between statin users and nonusers: ${ }^{\mathrm{a}} p<0.05 ;{ }^{\mathrm{b}} p<0.01$; ${ }^{\mathrm{c}} p<0.001$.

but also through anti-inflammatory effects. ${ }^{26}$ Results from the JUPITER trial demonstrated that the risk of cardiovascular events decreased by $79 \%$ in rosuvastatin users who achieved the targets of high-sensitivity C-reactive protein less than $1 \mathrm{mg} / \mathrm{L}$ and LDL cholesterol less than $1.8 \mathrm{mmol} / \mathrm{L}(\mathrm{HR}=0.21$, 95\% CI: 0.09-0.51). This effect was less prominent in patients who achieved only the target of LDL cholesterol ( $H R=0.49,95 \%$ $\mathrm{CI}$ : 0.37-0.66). These results suggest that the anti-inflammatory effects are a part of the complex mechanism by which statins exercise their activities on cardiovascular events and probably on VTE events. ${ }^{27,28}$

The RIETE registry provides data on the treatment of VTE in the real world with unselected population. Data from our patients reflect routine, unmonitored medical practice involving a broad spectrum of patients with VTE. It can, therefore, provide insights into the natural history of VTE in the special subgroup populations and to be hypothesis generating. The same reasons may result in some limitations. First, RIETE is an observational study with no randomization to statins versus no statins users, although the number of patients included was high. Thus, some nonmeasured variables, such as the reason why patients were using statins, could lead to a possible bias. Second, residual confounding may remain in the findings, as certain potential confounding variables may not be recorded on the database or may not be recorded in sufficient detail to completely remove their confounding effect. Third, in RIETE, we do not gather information on the use of statins over time, but only at baseline. However, since anticoagulant therapy is not a contraindication to stop the use of statins, we may assume (with limitations) that most patients did continue with this therapy. Fourth, uncontrolled healthy-user or healthy-adherer bias cannot be excluded either. But statin users in our cohort were 10 years older and had more comorbidities than nonusers. Although we provided adjusted results for several of the outcomes, we cannot exclude the possibility of residual differences in the two groups, and hence residual confounding. Fifth, we cannot be excluded a "social effect" where statin users may have a better health care. Finally, almost half of the mortality is cancer related ( $\mathbf{- T a b l e ~} \mathbf{3}$ ), and this drives 
e242 All-Cause Mortality during Anticoagulation for VTE Siniscalchi et al.

Table 4 Clinical outcomes during anticoagulation, according to the type of statin

\begin{tabular}{|c|c|c|c|c|c|c|c|c|}
\hline & \multicolumn{2}{|r|}{ Simvastatin } & \multicolumn{2}{|r|}{ Atorvastatin } & \multicolumn{2}{|r|}{ Rosuvastatin } & \multicolumn{2}{|r|}{ Others } \\
\hline & $n$ & $\begin{array}{l}\text { Events per } \\
100 \text { patient-years }\end{array}$ & $n$ & $\begin{array}{l}\text { Events per } \\
100 \text { patient-years }\end{array}$ & $n$ & $\begin{array}{l}\text { Events per } \\
100 \text { patient-years }\end{array}$ & $n$ & $\begin{array}{l}\text { Events per } \\
100 \text { patient-years }\end{array}$ \\
\hline Patients $(n)$ & \multicolumn{2}{|c|}{1,406} & \multicolumn{2}{|c|}{1,328} & \multicolumn{2}{|c|}{246} & \multicolumn{2}{|c|}{1,085} \\
\hline Recurrent DVT & 20 & $1.67(1.05-2.54)$ & 14 & $1.38(0.78-2.25)$ & 2 & $1.09(0.18-3.61)$ & 17 & $1.68(1.01-2.63)$ \\
\hline Recurrent PE & 17 & $1.41(0.85-2.21)$ & 16 & $1.58(0.94-2.52)$ & 2 & $1.09(0.18-3.61)$ & 11 & $1.07(0.56-1.85)$ \\
\hline Recurrent VTE & 36 & $3.03(2.16-4.15)$ & 28 & $2.79(1.89-3.98)$ & 4 & $2.19(0.70-5.29)$ & 27 & $2.68(1.80-3.85)$ \\
\hline Major bleeding & 36 & $3.00(2.13-4.11)$ & 53 & $5.19(3.93-6.74)^{b}$ & 14 & $7.93(4.52-13.0)^{b}$ & 33 & $3.20(2.24-4.44)$ \\
\hline Death & 92 & $7.56(6.13-9.23)^{c}$ & 114 & $11.1(9.16-13.2)$ & 23 & $12.5(8.12-18.5)$ & 71 & $6.82(5.37-8.56)^{c}$ \\
\hline \multicolumn{9}{|l|}{ Causes of death } \\
\hline Pulmonary embolism & 3 & $0.25(0.06-0.67)$ & 7 & $0.68(0.30-1.34)$ & 1 & $0.54(0.03-2.68)$ & 3 & $0.29(0.07-0.78)$ \\
\hline Initial PE & 1 & $0.08(0.00-0.41)^{a}$ & 6 & $0.58(0.24-1.21)$ & 1 & $0.54(0.03-2.68)$ & 3 & $0.29(0.07-0.78)$ \\
\hline Recurrent PE & 2 & $0.16(0.03-0.54)$ & 1 & $0.10(0.00-0.48)$ & 0 & - & 0 & - \\
\hline Bleeding & 2 & $0.16(0.03-0.54)^{a}$ & 8 & $0.78(0.36-1.47)$ & 1 & $0.54(0.03-2.68)$ & 7 & $0.67(0.29-1.33)$ \\
\hline Respiratory failure & 7 & $0.58(0.25-1.14)$ & 5 & $0.48(0.18-1.07)$ & 1 & $0.54(0.03-2.68)$ & 3 & $0.29(0.07-0.78)$ \\
\hline Sudden, unexpected & 3 & $0.25(0.06-0.67)$ & 4 & $0.39(0.12-0.94)$ & 0 & - & 0 & - \\
\hline Malignancy & 47 & $3.86(2.87-5.09)$ & 38 & $3.69(2.65-5.01)$ & 11 & $5.98(3.15-10.4)$ & 29 & $2.79(1.90-3.95)^{\mathrm{a}}$ \\
\hline Infection & 11 & $0.90(0.48-1.57)$ & 9 & $0.87(0.43-1.60)$ & 0 & - & 2 & $0.19(0.03-0.64)^{a}$ \\
\hline Multiorganic failure & 1 & $0.08(0.00-0.41)^{\mathrm{b}}$ & 8 & $0.78(0.36-1.47)$ & 3 & $1.63(0.42-4.44)$ & 5 & $0.48(0.18-1.07)$ \\
\hline Heart failure & 1 & $0.08(0.00-0.41)$ & 9 & $0.87(0.43-1.60)$ & 1 & $0.54(0.03-2.68)$ & 4 & $0.38(0.12-0.93)$ \\
\hline Bronchoaspiration & 0 & - & 3 & $0.29(0.07-0.79)$ & 0 & - & 3 & $0.29(0.07-0.78)$ \\
\hline Ischemic stroke & 0 & - & 3 & $0.29(0.07-0.79)$ & 0 & - & 1 & $0.10(0.00-0.47)$ \\
\hline Myocardial infarction & 0 & - & 1 & $0.10(0.00-0.48)$ & 0 & - & 1 & $0.10(0.00-0.47)$ \\
\hline
\end{tabular}

Abbreviations: DVT, deep vein thrombosis; PE, pulmonary embolism; VTE, venous thromboembolism.

Notes: Results expressed as number of events per 100 patient-years.

Differences between subgroups: ${ }^{\mathrm{a}} p<0.05$; ${ }^{\mathrm{b}} p<0.01$; ${ }^{\mathrm{c}} p<0.001$.

Table 5 Multivariable analyses for all-cause death

\begin{tabular}{|l|l|l|}
\hline & Univariable & Multivariable \\
\hline & 1,632 & \\
\hline Statins users (yes) & $0.82(0.73-0.93)^{\mathrm{a}}$ & $0.68(0.59-0.79)^{\mathrm{b}}$ \\
\hline Nonusers & Ref. $^{\mathrm{a}}$ & Ref. $^{\mathrm{b}}$ \\
\hline Simvastatin & $0.72(0.58-0.88)^{\mathrm{a}}$ & $0.64(0.52-0.80)^{\mathrm{b}}$ \\
\hline Atorvastatin & $1.00(0.83-1.21)$ & $0.72(0.58-0.89)^{\mathrm{a}}$ \\
\hline Rosuvastatin & $1.10(0.73-1.66)$ & $0.77(0.50-1.19)$ \\
\hline Others & $0.70(0.55-0.89)^{\mathrm{a}}$ & $0.67(0.52-0.87)^{\mathrm{a}}$ \\
\hline
\end{tabular}

Notes: Results are expressed as hazard ratio (95\% confidence intervals). Variables entering in the multivariate analysis: patients' age, body weight, chronic lung disease, chronic heart failure, recent major bleeding, recent surgery, recent immobility $\geq 4$ days, estrogen use, pregnancy, active cancer, prior VTE, initial VTE presentation, anemia, platelet count $<100,000 / \mu \mathrm{L}$, creatinine clearance levels, diabetes, current smoking, prior myocardial infarction, prior ischemic stroke, peripheral artery disease, concomitant therapy with antiplatelet drugs, and concomitant therapy with corticosteroids.

${ }^{\mathrm{a}} \mathrm{p}<0.01$.

${ }^{\mathrm{b}} \mathrm{p}<0.001$.

the reduced mortality in users. In contrast, cardiovascular mortality (stroke, myocardial infraction, and heart failure) is rather low. Hence, if there is a causal impact of statins on

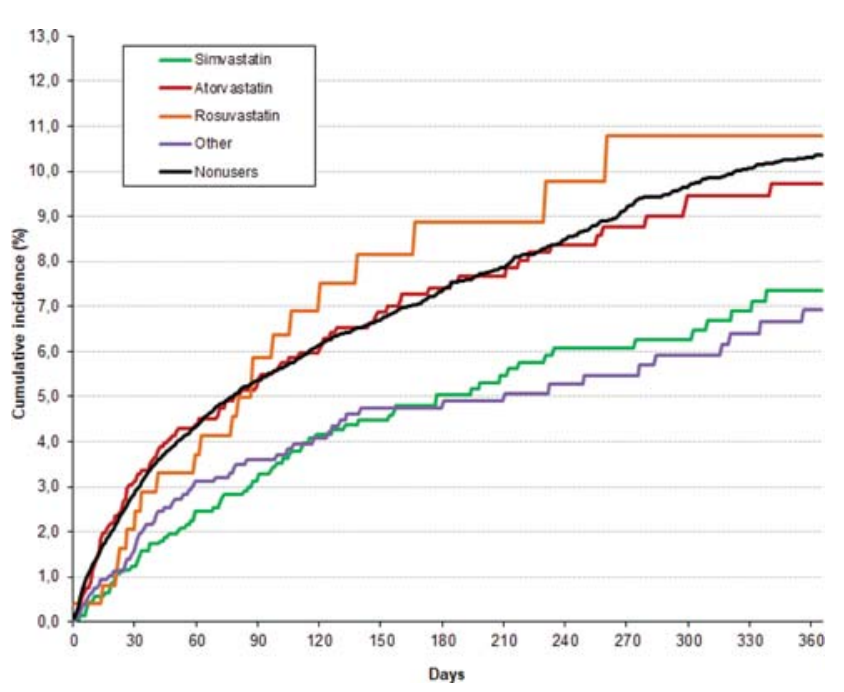

Fig. 2 Cumulative mortality rates according to type of statin used.

mortality in VTE patients, this is unlikely to be explained by major adverse cardiovascular events.

\section{Conclusion}

In conclusion, our findings confirm that in patients receiving anticoagulant therapy for VTE the use of statins was 
associated with a $20 \%$ decrease in mortality, irrespective of different statin type. Intervention studies specifically designed to confirm our findings in patients receiving anticoagulant therapy for VTE are warranted.

\section{Note}

Coordinator of the RIETE Registry: M.M.

RIETE steering committee members: Paolo Prandoni, Benjamin Brenner, and D.F.-B.

RIETE national coordinators: Raquel Barba (Spain), Pierpaolo Di Micco (Italy), Laurent Bertoletti (France), Sebastian Schellong (Germany), Inna Tzoran (Israel), Abilio Reis (Portugal), Marijan Bosevski (R. Macedonia), Henri Bounameaux (Switzerland), Radovan Malý (Czech Republic), Peter Verhamme (Belgium), Joseph A. Caprini (USA), and Hanh My Bui (Vietnam).

RIETE Registry coordinating center: S\&H Medical Science Service.

\section{Funding}

We express our gratitude to Sanofi Spain for supporting this Registry with an unrestricted educational grant.

\section{Conflict of Interest}

C.S. received speaker's fee for congress presentation by MediK. C.G.-C. reports nonfinancial support from Leo Pharma, nonfinancial support from Leo Pharma, nonfinancial support from Sanofi-Aventis, outside the submitted work; C.G.-C. reports nonfinancial support from Leo Pharma, nonfinancial support from Sanofi-Aventis, outside the submitted work.

\section{Acknowledgments}

We also thank the RIETE Registry coordinating center, S\&H Medical Science Service, for their quality control data, logistic, and administrative support and Prof. Salvador Ortiz, Universidad Autónoma Madrid and Silvia Galindo, both Statistical Advisors in S\&H Medical Science Service, for the statistical analysis of the data presented in this paper.

\section{References}

1 Hong KS, Lee JS. Statins in acute ischemic stroke: a systematic review. J Stroke 2015;17(03):282-301

2 Ní Chróinín D, Asplund K, Åsberg S, et al. Statin therapy and outcome after ischemic stroke: systematic review and metaanalysis of observational studies and randomized trials. Stroke 2013;44(02):448-456

3 Szummer K, Lundman P, Jacobson SH, et al. Association between statin treatment and outcome in relation to renal function in survivors of myocardial infarction. Kidney Int 2011;79(09): 997-1004

4 Tonelli M, Moyé L, Sacks FM, Kiberd B, Curhan GCholesterol and Recurrent Events (CARE) Trial Investigators. Pravastatin for secondary prevention of cardiovascular events in persons with mild chronic renal insufficiency. Ann Intern Med 2003; 138(02):98-104

5 Zhang X, Jing J, Zhao X, et al. Statin use during hospitalization and short-term mortality in acute ischaemic stroke with chronic kidney disease. Eur Neurol 2018;79(5,6):296-302
6 Oesterle A, Laufs U, Liao JK. Pleiotropic effects of statins on the cardiovascular system. Circ Res 2017;120(01):229-243

7 Glynn RJ, Danielson E, Fonseca FAH, et al. A randomized trial of rosuvastatin in the prevention of venous thromboembolism. $\mathrm{N}$ Engl J Med 2009;360(18):1851-1861

8 Zaccardi F, Kunutsor SK, Seidu S, Davies MJ, Khunti K. Is the lower risk of venous thromboembolism with statins related to lowdensity-lipoprotein reduction? A network meta-analysis and meta-regression of randomised controlled trials. Atherosclerosis 2018;271:223-231

9 Orsi FA, Biedermann JS, Kruip MJHA, et al. Rosuvastatin use reduces thrombin generation potential in patients with venous thromboembolism: a randomized controlled trial. J Thromb Haemost 2019;17(02):319-328

10 Biere-Rafi S, Hutten BA, Squizzato A, et al. Statin treatment and the risk of recurrent pulmonary embolism. Eur Heart J 2013;34 (24):1800-1806

11 Schmidt M, Cannegieter SC, Johannesdottir SA, Dekkers OM, Horváth-Puhó E, Sørensen HT. Statin use and venous thromboembolism recurrence: a combined nationwide cohort and nested case-control study. J Thromb Haemost 2014;12(08):1207-1215

12 Siniscalchi C, Quintavalla R, Rocci A, et al; RIETE Investigators. Statin and all-cause mortality in patients receiving anticoagulant therapy for venous thromboembolism. Data from the RIETE registry. Eur J Intern Med 2019;68:30-35

13 Muriel A, Jiménez D, Aujesky D, et al; RIETE Investigators. Survival effects of inferior vena cava filter in patients with acute symptomatic venous thromboembolism and a significant bleeding risk. J Am Coll Cardiol 2014;63(16):1675-1683

14 Farge D, Trujillo-Santos J, Debourdeau P, et al; RIETE Investigators. Fatal events in cancer patients receiving anticoagulant therapy for venous thromboembolism. Medicine (Baltimore) 2015;94(32):e1235

15 Jiménez D, de Miguel-Díez J, Guijarro R, et al; RIETE Investigators. Trends in the management and outcomes of acute pulmonary embolism. Analysis from the RIETE Registry. J Am Coll Cardiol 2016;67(02):162-170

16 Muñoz-Torrero JFS, Bounameaux H, Pedrajas JM, et al; Registro Informatizado de la Enfermedad TromboEmbólica (RIETE) Investigators. Effects of age on the risk of dying from pulmonary embolism or bleeding during treatment of deep vein thrombosis. J Vasc Surg 2011;54(6, suppl)26S-32S

17 Moustafa F, Pesavento R, di Micco P, et al; RIETE Investigators. Real-life use of anticoagulants in venous thromboembolism with a focus on patients with exclusion criteria for direct oral anticoagulants. Clin Pharmacol Ther 2018;103(04):684-691

18 Monreal M, Kakkar AK, Caprini JA, et al; RIETE Investigators. The outcome after treatment of venous thromboembolism is different in surgical and acutely ill medical patients. Findings from the RIETE registry. J Thromb Haemost 2004;2(11):1892-1898

19 Bikdeli B, Jimenez D, Hawkins M, et al; RIETE Investigators. Rationale, design and methodology of the computerized registry of patients with venous thromboembolism (RIETE). Thromb Haemost 2018;118(01):214-224

20 Stone NJ, Robinson JG, Lichtenstein AH, et al; American College of Cardiology/American Heart Association Task Force on Practice Guidelines. 2013 ACC/AHA guideline on the treatment of blood cholesterol to reduce atherosclerotic cardiovascular risk in adults: a report of the American College of Cardiology/American Heart Association Task Force on Practice Guidelines. J Am Coll Cardiol 2014;63(25 pt B):2889-2934

21 Biedermann JS, Kruip MJHA, van der Meer FJ, et al. Rosuvastatin use improves measures of coagulation in patients with venous thrombosis. Eur Heart J 2018;39(19):1740-1747

22 Tripodi A, Legnani C, Chantarangkul V, Cosmi B, Palareti G, Mannucci PM. High thrombin generation measured in the presence of thrombomodulin is associated with an increased risk of recurrent venous thromboembolism. J Thromb Haemost 2008;6 (08):1327-1333 
e244 All-Cause Mortality during Anticoagulation for VTE Siniscalchi et al.

23 Szczeklik A, Musiał J, Undas A, et al. Inhibition of thrombin generation by simvastatin and lack of additive effects of aspirin in patients with marked hypercholesterolemia. J Am Coll Cardiol 1999;33(05):1286-1293

24 Cortellaro M, Cofrancesco E, Arbustini E, et al. Atorvastatin and thrombogenicity of the carotid atherosclerotic plaque: the ATROCAP study. Thromb Haemost 2002;88(01):41-47

25 Macchia A, Laffaye N, Comignani PD, et al. Statins but not aspirin reduce thrombotic risk assessed by thrombin generation in diabetic patients without cardiovascular events: the RATIONAL trial. PLoS One 2012;7(03):e32894
26 Ural AU, Yilmaz MI, Avcu F, Yalcin A. Treatment with cerivastatin in primary mixed hyperlipidemia induces changes in platelet aggregation and coagulation system components. Int J Hematol 2002;76(03):279-283

27 Ridker PM, Danielson E, Fonseca FA, et al; JUPITER Trial Study Group. Reduction in C-reactive protein and LDL cholesterol and cardiovascular event rates after initiation of rosuvastatin: a prospective study of the JUPITER trial. Lancet 2009;373(9670):1175-1182

28 Kessinger CW, Kim JW, Henke PK, et al. Statins improve the resolution of established murine venous thrombosis: reductions in thrombus burden and vein wall scarring. PLoS One 2015;10(02):e0116621 